\title{
The quest of chirality in the interstellar medium
}

\section{Lessons of propylene oxide detection}

\author{
Y. Ellinger ${ }^{1}$, F. Pauzat ${ }^{1}$, A. Markovits ${ }^{1}$, A. Allaire ${ }^{1}$, and J.-C. Guillemin ${ }^{2}$ \\ ${ }^{1}$ Sorbonne Université, CNRS, Laboratoire de Chimie Théorique, LCT, 75005 Paris, France \\ e-mail: yves.ellinger@sorbonne-universite.fr \\ ${ }^{2}$ Univ. Rennes, Ecole Nationale Supérieure de Chimie de Rennes, CNRS, ISCR UMR6226, 35000 Rennes, France \\ e-mail: jean-claude.guillemin@ensc-rennes.fr
}

Received 11 October 2019 / Accepted 29 November 2019

\begin{abstract}
Context. All but one complex organic molecule (COM) detected so far in the interstellar medium (ISM) are achiral; propylene oxide $\left(\mathrm{c}-\mathrm{C}_{2} \mathrm{H}_{3} \mathrm{O}\right)-\mathrm{CH}_{3}$ is the only exception to this. Finding other chiral species is a priority for astrobiology to progress in the understanding of the emergence of life. Whatever the conditions of their formation, i.e., gas phase or grain chemistry, the detection relies on rotational spectra. This means that, if adsorbed after formation in the gas phase or directly formed on the icy grains, these COMs must escape in the gas phase as free flyers to be detectable.

Aims. Learning the lesson drawn from the only observation of a chiral compound and considering the structural constraints imposed to a molecule to be chiral, we look at what species could satisfy these conditions and be potential targets for a radio astronomy search in the ISM gas phase.

Methods. This question was addressed by combining two complementary approaches that rely on density functional theory. The structure, energetics, and spectroscopic parameters of each potential candidate were determined using molecular calculations. The propensity for a molecule to remain trapped on the ice coating of the grains was evaluated by numerical simulations making use of a solid state periodic model.

Results. Replacing the $-\mathrm{CH}_{3}$ group on rigid propylene oxide by $-\mathrm{CN},-\mathrm{CCH},-\mathrm{NH}_{2},-\mathrm{OH}$, or $-\mathrm{HCO}$ gives oxirane daughter molecules whose adsorption energies divide into two classes: below and above the adsorption energy of $\mathrm{H}_{2} \mathrm{O}$ on solid water-ice $\sim 13.5 \mathrm{kcal} \mathrm{mol}^{-1}$. Conclusions. The best chiral candidate would be a rigid molecule for an easier determination of its radio spectra. This molecule would be composed of a central carbon linked to one hydrogen and three different chemical groups as simple as possible. If not the most stable isomer, this candidate should be as close as possible on the energy scale, possess a significant dipole moment, and be less strongly attached to the ice than $\mathrm{H}_{2} \mathrm{O}$ itself.
\end{abstract}

Key words. ISM: abundances - astrobiology - solid state: volatile

\section{Introduction}

Over 210 different species (McGuire 2018) have been detected in the interstellar and circumstellar media (ISM). More than 50 of these complex organic molecules (COMs) are composed of at least 6 atoms and present the characteristic functional groups necessary to initiate a complex organic chemistry that could normally lead to chiral species. Only one chiral molecule, propylene oxide (also known as methyl oxirane), (cyc- $\left.\mathrm{C}_{2} \mathrm{H}_{3}-\mathrm{O}\right)-\mathrm{CH}_{3}$, has been identified in the ISM (McGuire et al. 2016), although several of these molecules larger than 10 atoms are common in carbonaceous chondrites (Martins \& Sephton 2009). It should be remembered that more than 70 amino acids, including 8 out of 21 present in living organisms (Cooper et al. 1992), were isolated in the Murchison meteorite.

In addition, the presence of an enantiomeric excess in organic matter has been found in the study of well-defined families of carbonaceous chondrites (Cronin \& Pizzarello 1997; Engel \& Macko 1997; Pizzarello \& Cronin 1998, 2000). Not only is the origin of this excess unknown, but the emergence itself of the first chiral molecule is still an unanswered question; no branched molecule has been identified so far in the ISM except isopropyl cyanide and propylene oxide. Identifying any chiral species other than propylene oxide in the ISM would be a considerable breakthrough.
Several reasons for the lack of detection come to mind: either the chiral species are produced inside the chondrites themselves according a process yet to be determined or these species are trapped on the surface of the icy grains and thus not present in the gas phase with an abundance large enough to be detected. Closely related to the last point is that we did not target the right molecules in the ISM search. The lack of additional detections may also be because of constraints on the detection techniques themselves, possibly for technical reasons, i.e., the difficulty of obtaining and analyzing ro-vibrational spectra in the laboratory that are precise enough to be matched with the observations. Defining appropriate criteria for selecting potential candidates is therefore of primordial importance. Before addressing the question of what molecules to search for, we note the imperative constraints imposed upon the structure of chiral species.

The first definition of chirality was given more than a century ago (Kelvin 1904). It was phrased as, "I call any geometrical figure, or group of points, chiral, and say it has chirality, if its image in a plane mirror, ideally realized, cannot be brought to coincide with itself".

Pasteur was the first to suggest that molecular dissymmetry may arise from a tetrahedral or helical arrangement of atoms. Presented soon after, the tetrahedral carbon hypothesis of Le Bel and van't Hoff was a first rationalization of the discovery of molecular chirality on the tartaric acid salts. This tetrahedral 


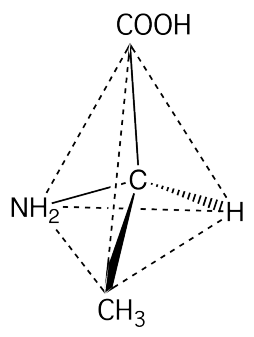

Alanine

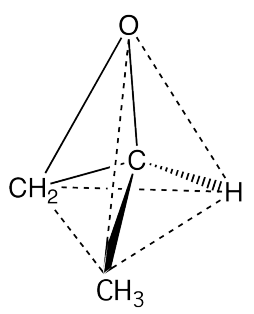

Propylene oxide
Fig. 1. Le Bel and van't Hoff stereochemical representations. The asymmetric carbon is at the center of the tetrahedron.

representation is presented in Fig. 1 as examples of the simplest chiral amino acid, alanine, and the first chiral molecule observed in the ISM, i.e., propylene oxide.

Strictly speaking, the smallest chiral molecules to be identified should be those with a deuterium atom on the asymmetric center. These are most probably $\mathrm{CH}_{3}-\mathrm{CHD}-\mathrm{OH}$ or $\mathrm{CH}_{3}-\mathrm{CHD}$ $\mathrm{CN}$ in view of the abundance of the parent species in hot media, but we could easily think of other candidates such as cyc- $\mathrm{CH}_{2}-$ $\mathrm{O}-\mathrm{CHD}$. Under these conditions, the obvious choice is a carbon atom linked to four different substituents. To be chemically significant, the molecule cannot be reduced to isotopic substitutions of $\mathrm{FCH}_{3}$ such as FCHDT. This molecule cannot also be limited to substitutions by halogens because a molecule such as HCF$\mathrm{BrCl}$ is totally unrealistic knowing the abundance of halogens in the ISM.

All the theoretical data reported in this work were obtained by means of quantum numerical simulations using standard approaches for both free flyer structures and energetics and for solid gas interactions. All technical details on the methods used are given in the appendix.

\section{Lesson from propylene oxide detection}

The fact that there is only one chiral molecule observed today should not be considered as an accident. Several plausible reasons, electronic as well as structural, could explain that so many attempts to detect chiral prebiotic species have failed while propylene oxide was observed. In contrast, it is possible to quote some specific characteristics of propylene oxide and its isomers, including their adsorption energies on the ice $E_{\text {Ads }}$ (see Table 1) that might have led to successful detection:

(i) Propylene oxide is a small molecule. Discarding molecules whose chirality stems from isotope or halogen substitutions, propylene oxide is one of the simplest chiral structures of prebiotic interest with only 10 atoms.

(ii) Propylene oxide is a "rigid molecule". Excepting the rotation of the $-\mathrm{CH}_{3}$ substituent, all movements are well-restricted bond length stretchings and angle deformations, thereby making the analysis of the spectra in the laboratory easier.

(iii) Propylene oxide has a significant dipole moment. The line intensities in rotational spectra scale with the square of $\mu$. With a value of $\sim 2$ Debye, propylene oxide is suitable for radio detection. This value is similar to those of its $\mathrm{C}_{3} \mathrm{H}_{6} \mathrm{O}$ isomers already detected in the ISM, i.e., acetone and propanal ( $\sim 3.0$ and 2.8 Debye, respectively; Combes et al. 1987; Snyder et al. 2002; Hollis et al. 2004).

(iv) Propylene oxide is abundant enough to be observed. The fact that $\sim 90 \%$ of the simple molecules identified (McGuire 2018) are the most stable isomers of a chemical formula is a
Table 1. Computed relative stabilities and energy ranking with respect to the isomer of minimum energy of same formula $\Delta E^{(a)}\left(\mathrm{kcal} \mathrm{mol}^{-1}\right)$, dipole moments $\mu^{(a)}$ (Debye), and adsorption energies of detected molecules on water ice $E_{\mathrm{Ads}}{ }^{(b)}\left(\mathrm{kcal} \mathrm{mol}^{-1}\right)$ of chiral $(*)$ propylene oxide and low energy isomers.

\begin{tabular}{|c|c|c|c|c|c|}
\hline Ranking & Isomer & $\Delta E$ & $\mu$ & $E_{\mathrm{Ads}}$ & Spectra \\
\hline 1 & & $0.0^{\mathrm{Obs}}$ & 3.1 & 6.0 & Mil \\
\hline 2 & & $7.1^{\mathrm{Obs}}$ & 2.8 & 11.6 & Mil \\
\hline 3 & & 11.7 & 0.5 & & NA \\
\hline 4 & & 15.9 & 1.4 & & NA \\
\hline 5 & & 21.9 & 1.6 & & Mil \\
\hline 6 & & 28.0 & 1.5 & & MW \\
\hline 7 & & 28.6 & 1.7 & & Mil \\
\hline 8 & & $29.9^{*, \mathrm{Obs}}$ & 2.0 & 10.6 & Mil \\
\hline
\end{tabular}

Notes. Spectral signatures are noted MW (microwave) and Mil (millimetric) or NA (not available). The molecules identified in the ISM are noted (Obs). ${ }^{(a)}$ Energy differences and dipole moments taken from Karton \& Talbi (2014); ${ }^{(b)}$ this work.

References. Corresponding to ranking orders (1) Groner et al. (2008);

(2) Zingsheim et al. (2017); (3) not available; (4) not available; (5) Melandri et al. (1994); (6) McDonald et al. (1978); (7) Daly et al. (2014); (8) Mesko et al. (2017).

pragmatic hint of the possibility of detection of new molecules. Relying on what is called the minimum energy principle (MEP; Lattelais et al. 2009, 2010a) possible chiral targets should be the most stable isomers or, if not, close in energy since the higher the energy of the target molecule, the lower might be the abundance relative to that of the most stable compound. The case of propylene oxide, $\sim 30 \mathrm{kcal} \mathrm{mol}^{-1}$ above acetone, which is equally close to that of oxirane above acetaldehyde, gives at least an idea of the order of magnitude of the energy gap allowing a plausible detection.

(v) Propylene oxide is a free flyer. It is well known that adsorption of molecules on icy surfaces is a crucial parameter to experimental observations in the laboratory and in space. In the laboratory for example, temperature programmed desorption (TPD) experiments have identified (Lattelais et al. 2011) dimethyl ether and methyl formate; they were able to determine adsorption energies of $E_{\mathrm{Ads}}=8.1 \pm 1.0$ and $8.9 \pm$ $1.0 \mathrm{kcal} \mathrm{mol}^{-1}$ comparable to the theoretical values of $E_{\mathrm{Ads}}=8.4$ and $9.2 \mathrm{kcal} \mathrm{mol}^{-1}$ respectively. Neither ethanol nor acetic acid could be identified in these low temperature TPD experiments. Such isomers are mainly detected in warm regions (Remijan et al. 2002; Requena-Torres et al. 2006), but even in these regions their abundances are well below those of their isomers of the same generic formula. The abundances ratios ethanol/dimethyl ether $\sim 0.3-3$ and acetic acid/methyl formate $\sim 0.01-0.1$ are not 
what they should be according the MEP since they are the most stable species in their respective $\mathrm{C}_{2} \mathrm{H}_{6} \mathrm{O}$ and $\mathrm{C}_{2} \mathrm{H}_{4} \mathrm{O}_{2}$ series. It is worth noting that the adsorption energies of $E_{\mathrm{Ads}}=13.5$ and $16.2 \mathrm{kcal} \mathrm{mol}^{-1}$ for ethanol and acetic acid, respectively, are of the same order of magnitude or larger than that of $\mathrm{H}_{2} \mathrm{O}$ on the ice. This might explain the reason for their non-characterization in the TPD experiments since these species cannot be ejected in the gas phase before the ice support (Lattelais et al. 2011). This would also be compatible with an abundance in the cold media that is too low to be detected with the present instruments.

At this point we note that microwave/millimetric spectra used to probe the presence and relative abundances of gas-phase species cannot provide the number of molecules effectively formed, but can only count those molecules that escape trapping on the surface of the icy grains. Whether it is a free flyer or not a free flyer, the key parameter is the adsorption energy on water ice. In other words, it can be conjectured that not detecting a molecule does not mean that it is not present in the ISM.

A last but trivial reason for a non-detection of all isomers of lower energy in a given series can be seen from the last column of Table 1. Not all the necessary spectral information are available to attempt a search for all compounds (for example isomers 3 and 4).

Propylene oxide is found in eighth position on the energy scale above acetone (Table 1). The five isomers below in energy have either an $-\mathrm{OH}$ function available to form hydrogen bonds with the ice surface or a $\mathrm{C}=\mathrm{C}$-O- group with delocalized electrons ready for van der Waals interactions. All these species, strongly attached to the surface, should not desorb easily before ice and thus be hardly detectable. By contrast, propylene oxide has only one oxygen atom capable, a priori, to form a hydrogen bond with dangling $\mathrm{H}$ atoms belonging to the ice surface, but no $\mathrm{OH}$ bond to link with an oxygen of the ice. This implies a rather low attachment energy to the ice and therefore, a better chance to turn into a free flyer.

Related species, with a single and accessible oxygen atom, have been already detected (therefore free flyers) such as acetone (Combes et al. 1987; Snyder et al. 2002), propanal (Hollis et al. 2004), dimethyl ether (Snyder et al. 1974), and oxirane (Dickens et al. 1997; Nummelin et al. 1998a,b). A recent survey toward the low-mass protostar IRAS 16293-2422 reported recently the first simultaneous detections of oxirane, acetone, and propanal (Lykke et al. 2017). It is worth noting that the relative abundance ratios of the two sets of isomers, a $\mathrm{CH}_{3} \mathrm{COCH}_{3} / \mathrm{C}_{2} \mathrm{H}_{5} \mathrm{CHO}$ ratio of 8 and a $\mathrm{CH}_{3} \mathrm{CHO} / \mathrm{c}-\mathrm{C}_{2} \mathrm{H}_{4} \mathrm{O}$ ratio of 12 reported by Lykke et al. (2017), are well in line with previous observations toward highmass protostars (and consistent with MEP). Furthermore, the adsorption energies of these species in the range 6-11 kcal mol${ }^{-1}$ are all below the binding energy of $\mathrm{H}_{2} \mathrm{O}$ on solid water (13$14 \mathrm{kcal} \mathrm{mol}^{-1}$ ), which is also consistent with the fact that these species are free flyers.

\section{Constraints on potential targets: propylene oxide siblings}

The main idea is to mimic propylene oxide, keeping the rigidity of the three membered ring and replacing the methyl group by a chemical function frequently detected in the ISM. To limit the complexity of the spectra only two and three atom substituents were considered, namely, $-\mathrm{C} \equiv \mathrm{N},-\mathrm{OH}$, and $-\mathrm{NH}_{2},-\mathrm{C} \equiv \mathrm{CH},-\mathrm{CHO}$. All targets are composite systems including a rigid part in the form of a three membered oxiranyl ring attached to a substituent with fewer atoms than $-\mathrm{CH}_{3}$. Taking advantage of the actual
Table 2. Computed relative stabilities with respect to the minimum energy isomer in each series $\Delta E\left(\mathrm{kcal} \mathrm{mol}^{-1}\right)$, dipole moments $\mu$ (Debye), and adsorption energies $E_{\mathrm{Ads}}$ of oxirane and derivatives $\left(\mathrm{kcal} \mathrm{mol}^{-1}\right)$ on water ice.

\begin{tabular}{|c|c|c|c|c|}
\hline Isomer & Structure & $\Delta E$ & $\mu$ & $E_{\mathrm{Ads}}$ \\
\hline Oxirane & & 27.5 & 1.9 & 8.0 \\
\hline Propylene oxide & & 29.9 & 2.0 & 10.6 \\
\hline Hydroxyoxirane & & 47.2 & 1.6 & 19.7 \\
\hline Cyanooxirane & & 27.6 & 3.8 & 10.7 \\
\hline Aminooxirane & & 47.6 & 1.0 & 15.4 \\
\hline Ethynyloxirane & & 54.5 & 1.8 & 12.8 \\
\hline Formyloxirane & & 40.7 & 2.6 & 16.4 \\
\hline
\end{tabular}

knowledge of the propylene oxide spectrum should help in the analysis of laboratory data.

The reference compound chosen to center the discussion based on the data collected in Table 2 is the unsubstituted oxirane, $\mathrm{c}-\mathrm{C}_{2} \mathrm{H}_{4} \mathrm{O}$, whose detection relied on the millimeter data by Hirose (1974). This simple heterocycle ( 7 atoms) was first identified (Dickens et al. 1997; Nummelin et al. 1998a,b) about $20 \mathrm{yr}$ after the detection of the minimum energy isomer, acetaldehyde (Gottlieb 1973). It is about $30 \mathrm{kcal} \mathrm{mol}^{-1}$ higher than acetaldehyde on the energy scale with a dipole moment of 1.9 suitable to radio detection. The adsorption energy of oxirane on the ice is calculated to be $10.6 \mathrm{kcal} \mathrm{mol}^{-1}$, that is notably less than that of $\mathrm{H}_{2} \mathrm{O}$ on the same surface $\sim 13-14 \mathrm{kcal} \mathrm{mol}^{-1}$ ).

The first discovery of propylene oxide (McGuire et al. 2016) dates approximately $20 \mathrm{yr}$ after oxirane. Seeking simplicity, we compare all data in light of the lessons drawn from oxirane and propylene oxide.

\subsection{Structure and stability of molecular targets}

The first point to be noted (Table 2) is that none of these species are the minimum on the energy scale of the related isomers. The corresponding most stable molecules are as follows:

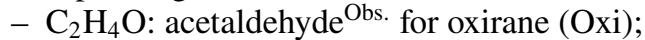

$-\mathrm{C}_{3} \mathrm{H}_{6} \mathrm{O}$ : acetone ${ }^{\mathrm{Obs}}$ for propylene oxide $(\mathrm{Oxi}-\mathrm{Me})$;

$-\mathrm{C}_{3} \mathrm{H}_{3} \mathrm{NO}$ : acetylcyanide for cyanooxirane (Oxi-CN);

$-\mathrm{C}_{2} \mathrm{H}_{5} \mathrm{NO}$ : acetamide ${ }^{\mathrm{Obs}}$. for aminooxirane $\left(\mathrm{Oxi}-\mathrm{NH}_{2}\right)$;

$-\mathrm{C}_{4} \mathrm{H}_{4} \mathrm{O}$ : furan for ethynyloxirane $(\mathrm{Oxi}-\mathrm{CCH})$; and

$-\mathrm{C}_{3} \mathrm{H}_{4} \mathrm{O}_{2}$ : acrylic acid for formyloxirane (Oxi-HCO).

The energy gap, $\Delta E$, between the target and the most stable isomer is between $\sim 20$ and $60 \mathrm{kcal} \mathrm{mol}^{-1}$. Four out of seven of the aforementioned most stable isomers (noted Isomer ${ }^{\text {Obs. }}$ ) have been identified in the ISM in agreement with MEP, but no molecule has been detected so far in the $\mathrm{C}_{3} \mathrm{H}_{3} \mathrm{NO}, \mathrm{C}_{4} \mathrm{H}_{4} \mathrm{O}$, and $\mathrm{C}_{3} \mathrm{H}_{4} \mathrm{O}_{2}$ series; even furan was not detected despite its aromatic electronic structure (Lattelais et al. 2010b). 
It should be remarked that when the most stable isomer has been observed, higher energy species have also been identified, although always later than the first isomer. In the $\mathrm{C}_{3} \mathrm{H}_{6} \mathrm{O}, \mathrm{C}_{2} \mathrm{H}_{4} \mathrm{O}_{2}$ and $\mathrm{C}_{2} \mathrm{H}_{5} \mathrm{NO}$ series propanal $\left(\mathrm{CH}_{3} \mathrm{CH}_{2} \mathrm{CHO}\right)$ at $7.1 \mathrm{kcal} \mathrm{mol}^{-1}$ above acetone, methylformate $\left(\mathrm{HCO}_{2} \mathrm{CH}_{3}\right)$ and glycolaldehyde $\left(\mathrm{HCOCH}_{2} \mathrm{OH}\right)$ at 17.2 and $27.4 \mathrm{kcal} \mathrm{mol}^{-1}$ above acetic acid, and N-methylformamide at $10.1 \mathrm{kcal} \mathrm{mol}^{-1}$ above acetamide have been identified, respectively.

\subsection{Target visibility and dipole moments}

A crucial parameter for the detection by microwave or millimetric spectroscopy is the dipole moment of the target. The dipole moment of the molecules already observed are comprised between 1.8 and 3.9 Debye as follows:

- Acetaldehyde: $\mu=2.9 \mathrm{D}$; oxirane: $\mu=1.9 \mathrm{D}$; vinyl alcohol: $\mu=1.0 \mathrm{D}$.

- Acetone: $\mu=3.1 \mathrm{D}$; propanal: $\mu=2.8 \mathrm{D}$; propylene oxide: $\mu=2.0 \mathrm{D}$.

- Acetic acid $\mu=1.8 \mathrm{D}$; methylformate $\mu=1.9 \mathrm{D}$; glycolaldehyde $\mu=2.5 \mathrm{D}$.

- Acetamide: $\mu=3.8 \mathrm{D}$; N-methylformamide: $\mu=3.9 \mathrm{D}$.

Since the dipole moments of the potential targets are all but one, in the range 1.8-3.8 Debye (see Table 2), this parameter should not be limiting for observation with the possible exception of aminooxirane for which $\mu=1.0 \mathrm{D}$ could be not large enough if associated with a small abundance. We can argue that vinyl alcohol has been detected in spite of a small dipole moment, but this is counterbalanced by a significant abundance (Turner \& Apponi 2001), which is even more true for propene, $(\mu=0.4 \mathrm{D})$ and methoxymethanol $(\mu=0.2 \mathrm{D})$.

\subsection{Target visibility and adsorption}

The last but not the least parameter to consider is the adsorption energy that governs the ability of the target species to escape from the grain surface into the gas phase. Concerning this parameter, one point should be underlined: the adsorption energies that are known (measured and/or calculated) for the species detected so far in the $\mathrm{C}_{x} \mathrm{H}_{y} \mathrm{O}_{z}$ target series considered, namely, $\mathrm{C}_{2} \mathrm{H}_{4} \mathrm{O}, \mathrm{C}_{3} \mathrm{H}_{6} \mathrm{O}$, and $\mathrm{C}_{2} \mathrm{H}_{4} \mathrm{O}_{2}$, are all below the adsorption of $\mathrm{H}_{2} \mathrm{O}$ on solid water $\left(13-14 \mathrm{kcal} \mathrm{mol}^{-1}\right)$. These are in the range $8-11 \mathrm{kcal} \mathrm{mol}^{-1}$, which is consistent with the fact that these species are easily converted into free flyers.

While discussing each molecule in particular, we have to keep in mind that a most plausible reason for a molecule being free flyer can be seen by looking at the adsorption sites as a function for their chemical structures.

Coming back to the oxirane derivatives listed in Table 2, a comparison of the adsorption situation of the simplest heterocycle target with that of the most stable isomer, acetaldehyde, is instructive (see Fig. 2).

In these adsorption complexes, there is only one $\mathrm{OH}$ bond formed to insure stability. Acetaldehyde is attached to the ice surface by a single $\mathrm{OH}$ bond between the carbonyl oxygen and a dangling $\mathrm{H}$ atom of the surface. In oxirane (Oxi), the interaction with the ice surface also proceeds via an $\mathrm{OH}$ bond between the oxygen of the three-membered ring and a dangling $\mathrm{H}$ atom of the surface. The adsorption energies are $E_{\mathrm{Ads}}=5.9 \mathrm{kcal} \mathrm{mol}^{-1}$ and $E_{\mathrm{Ads}}=8.0 \mathrm{kcal} \mathrm{mol}^{-1}$, respectively.

In the simple example shown above there is only one $\mathrm{OH}$ bond formed to insure stability. However, six out of seven derivatives listed in Table 2 contain two different functional groups that
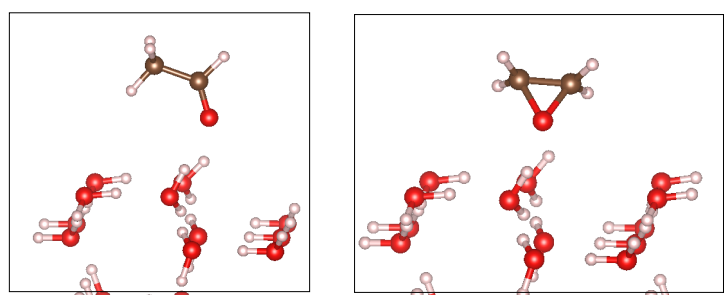

Fig. 2. Stereochemical representations of adsorption sites on the topmost ice bilayers. Observed most stable isomer: acetaldehyde (left); rigid target oxirane (right).

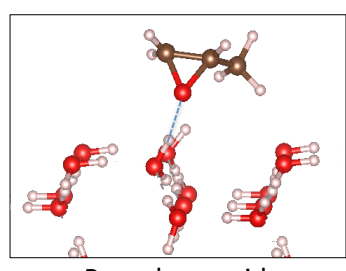

Propylene oxide
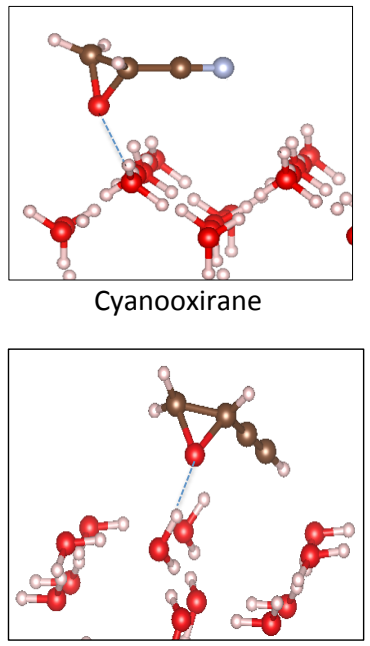

Ethynyloxirane

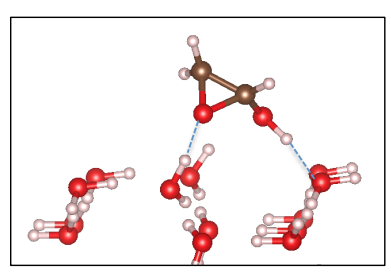

Hydroxyoxirane

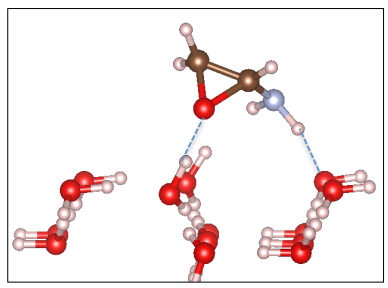

Aminooxirane

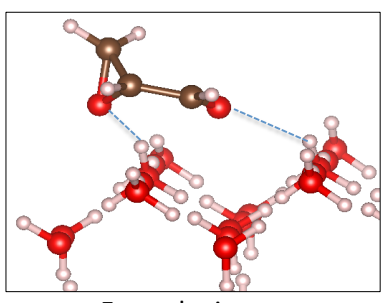

Formyloxirane
Fig. 3. Stereochemical representations of adsorption sites for oxirane based rigid targets on the ice surface.

might also more or less contribute to the adsorption energy as illustrated in Fig. 3.

(1) Propylene oxide (Oxi-Me), such as the simplest species (Oxi) in the oxirane series, has been detected using the millimeter-wave data by Creswell \& Schwendeman (1977), Daly et al. (2014). This species has only one strong point of attachment leading to the same type of $\mathrm{OH}$ bond to the ice support as oxirane. The methyl group is oriented almost parallel to the surface and does not form a strong second point of attachment with an oxygen of the ice. However, its influence cannot be totally neglected since the adsorption energy $E_{\mathrm{Ads}}$ is increased by $2.6 \mathrm{kcal} \mathrm{mol}^{-1}$ owing to the position of one $\mathrm{H}$ of $-\mathrm{CH}_{3}$ at $\sim 2.5 \AA$ of an oxygen of the surface. Me-Oxi and genuine Oxi belong to the same category with adsorption energies lower than that of $\mathrm{H}_{2} \mathrm{O}$ on ice and practically identical dipole moments, which is the reason they are both observed.

(2) Hydroxyoxirane (Oxi-OH) presents two oxygen atoms, which formally can be two possible attachment points to the surface. These two atoms are in a topological situation similar to that of acetic acid, i.e., well positioned to form the same kind of 
bridge with the ice surface (Lattelais et al. 2011). Unfortunately, to our knowledge, no spectrum seems available to start a search.

(3) Cyanooxirane (Oxi-CN) shows, a priori two possibilities for interacting with the ice, i.e., the oxiranyl oxygen and the nitrogen of the $-\mathrm{C} \equiv \mathrm{N}$ substituent. The value of the calculated $E_{\text {Ads }}=10.7 \mathrm{kcal} \mathrm{mol}^{-1}$ is only a little over that of Oxi-Me but higher than that of Oxi by $2.7 \mathrm{kcal} \mathrm{mol}^{-1}$. That the $-\mathrm{C} \equiv \mathrm{N}$ group does not increase $E_{\text {Ads }}$ significantly is a consequence of its orientation outward the ice surface. Spectroscopic data have been obtained for this species (Müller \& Bauder 1996; Behnke et al. 2004).

(4) Ethynyloxirane $(\mathrm{Oxi}-\mathrm{CCH})$ with a linear $-\mathrm{C} \equiv \mathrm{CH}$ group is structurally equivalent to $\mathrm{Oxi}-\mathrm{CN}$ and isoelectronic. It can reach at a longer distance and the acetylenic hydrogen may interact with an oxygen of the ice, whereas nitrogen would interact with a surface hydrogen. However this interaction is too weak to create an additional stabilization energy of $E_{\text {Ads }}\left(12.8 \mathrm{kcal} \mathrm{mol}^{-1}\right)$ that remains less than that of $\mathrm{H}_{2} \mathrm{O}$ on the ice or well below that of an alcoholic group such as in Oxi-OH. A similar conclusion had been drawn by Collins \& Boggs (1972) following their microwave study that indicated little effect of interaction between the acetylenic group and the ring.

(5) Aminooxirane $\left(\mathrm{Oxi}-\mathrm{NH}_{2}\right)$ is isoelectronic of Oxi-OH. The $\mathrm{NH}_{2}$ group provides a second point of attachment as $\mathrm{OH}$ does in Oxi-OH. The oxiranyl oxygen makes a hydrogen bond with a dangling $\mathrm{H}$ of the ice which, completed by the link between a $\mathrm{NH}$ bond with an oxygen of the ice, forms a strong bridge in the same way as Oxi-OH with the solid water. This species is another example for which spectroscopic data are missing to start a search.

(6) Formyloxirane (Oxi-CHO) presents two possible adsorption sites through two different oxygen atoms: one is common to all oxiranes, the other comes from the $>\mathrm{C}=\mathrm{O}$ aldehydic group. The two $\mathrm{OH}$ bonds formed are separated by a larger distance than previously allowing more flexibility of the structure and a better positioning over the surface. The resulting adsorption energy of $16.4 \mathrm{kcal} \mathrm{mol}^{-1}$ also conveys a situation that is a reminder of that of acetic acid and has a value of $E_{\text {Ads }}$ larger than the adsorption of $\mathrm{H}_{2} \mathrm{O}$ on the ice. Spectroscopic data are available (Creswell et al. 1977).

\section{Summary and concluding remarks}

Assuming that the detection of propylene oxide is not purely accidental, this report addresses the question of whether some chiral molecules have a probability of being detected in the ISM. To this end we tried to pinpoint the specificities of propylene oxide that would have made it suitable to radio detection, which means its presence in the gas phase of molecular clouds. In addition we considered also the energetic aspect, relying on the MEP, which states that the most abundant isomer of a given generic formula should be that of lowest energy.

The relative thermodynamic stability of the chiral molecules with respect to the other possible species of the same chemical formula is calculated by means of quantum simulations based on density functional theory (DFT). The ability of a given molecule to be free flyer in the ISM gas phase has been quantified by modeling its adsorption energy on the ice cover of interstellar grains by means of "first principle" solid state simulations compared to previous TPD experiments.

A number of molecules have been observed that are not the most stable, but it should be stressed that in most cases the lowest energy species of the family had already been observed. This is not a hopeless situation for molecules within $\sim 25-60 \mathrm{kcal} \mathrm{mol}^{-1}$ of the most stable isomer, provided their dipole moment is large enough since the radio line strength scales with the square of the dipole moment. Indeed, if none of the oxiranes is the minimum energy structure, even the two species already detected, the energy criterion should not be a prohibitive limiting issue, especially in cases in which isomers of the same series have already been detected. As a supporting example, we might cite the case of the manifold of $\mathrm{CHON}$ isomers, for which the most accurate $a b$ initio calculations have shown that, above the most stable $\mathrm{HNCO}$ isomer, $\mathrm{HOCN}$ and $\mathrm{HCNO}$ ranking at 24.7 and $70.7 \mathrm{kcal} \mathrm{mol}^{-1}$ on the energy scale have effectively been detected.

Another difficulty in the search for new molecules (chiral or not) is obtaining clean laboratory data to be matched with the observations. As of today, it is generally the lack of this spectroscopic information that likely limits the search for new species in space.

The series of oxirane-based derivatives fulfills the above structural and spectroscopic constraints, namely that of significant dipole moments and a priori that of an easier analysis of the radio spectra thanks to the rigid structure of the three membered ring and the actual knowledge of the spectroscopic data gathered along the detections of oxirane and propylene oxide.

Nevertheless, the necessity of the intended target to be a free flyer remains a major problem. Here we found two classes of oxiranes. On the one hand, we have the species with $E_{\text {Ads }}$ smaller than the adsorption of $\mathrm{H}_{2} \mathrm{O}$ on the ice, namely, oxirane, propylene oxide (already observed), and cyanooxirane; on the other hand, the species with $E_{\text {Ads }}$ larger than the adsorption of $\mathrm{H}_{2} \mathrm{O}$ on the ice, namely, hydroxyoxirane, aminoxirane, and formyloxirane.

The first type of molecules has only one point of attachment via an hydrogen bond to the surface; these molecules have a reasonable chance of being detected. The second type of molecules has two points of attachment via two hydrogen bonds; these molecules are much strongly tied to the ice surface and their detection seems more difficult. As for ethynyloxirane, it is an illustration of a situation "in between" and, even if the microwave spectrum has been obtained in the laboratory, the detection will remain a challenging issue. It should be emphasized that the above statements are pertinent to "cold" regions in the range 10-130 K below ice desorption. At higher temperatures when ice desorbs, two possibilities can be considered: either the target is pulled away with the sublimating ice or it diffuses to the subjacent refractory core where it remains attached until the temperature reaches a higher threshold characteristic of the deeper support $^{1}$.

In the end, three general criteria have been cleared that the target molecule should satisfy to be detected in an environment of icy grains. The target molecule should:

- have a simple structure, as rigid as possible, for an easier analysis of the microwave/millimetric signature;

- have a significant dipole moment to counterbalance a plausible low abundance;

- have an adsorption energy lower than that of $\mathrm{H}_{2} \mathrm{O}$ on ice to escape the grain surface.

A last question could be considered that remains unsettled in the present context, namely that of the formation of such compounds. The issue has been addressed recently for the case of propylene oxide by two different groups. Hudson et al. (2017) report experimental results of a low temperature formation of

1 This type of temperature selective adsorption will be discussed in a forthcoming report. 
propylene oxide following irradiation in analogs of interstellar ices. Bergantini et al. (2018) use the same approach, implying irradiation to trigger the formation of propylene oxide; in addition they perform quantum chemistry calculations in the gas phase to obtain information on exothermicities and activation barriers to be used in astrochemical modeling via a reaction network designed to account for the synthesis of the target compound in the ice. Both studies conclude that the main process at work at low temperatures is the reaction of what is referred to as suprathermal atomic oxygen onto the double bond of an unsaturated propene, i.e.,

$\mathrm{O}^{\star}+\mathrm{H}_{2} \mathrm{C}=\mathrm{CH}-\mathrm{CH}_{3} \rightarrow\left(\mathrm{c}-\mathrm{C}_{2} \mathrm{H}_{3} \mathrm{O}\right)-\mathrm{CH}_{3}$.

These authors point out that the role of ice is essential and that propylene oxide should be released one way or another in the gas phase to be detected. Our results are consistent with this double assertion. However, should this mechanism be general, a question they have left open, all chiral molecules discussed in this report would be potentially concerned by such a mechanism. Then, an additional constraint appears with the limitation of the abundance of the parent unsaturated organic compounds. As far as we know, only the parent molecules propene (for propylene oxide) and acrylonitrile (for cyanooxirane) are abundant species. Concerning the other supposedly potential parents, such as vinyl alcohol (for hydroxyoxirane), vinyl amine (for aminooxirane), butenyne (for ethynyloxirane), and propenal (for formyloxirane), the situation is different: either there is no detection at all or at best it is still under discussion.

Assembling all the criteria developed in the light of the present study leads us to propose cyanoxirane as the best chiral target to search for in the cold ISM. Ethynyloxirane is next if butenyne could be considered as potentially present.

As a final touch to this study of oxirane-based species, we should mention the simplest isotopomer, deuterated oxirane $\mathrm{c}-\mathrm{CH}_{2} \mathrm{CHDO}$, whose rotational spectrum has been analyzed recently (Albert et al. 2019). Its detection would be more than just a curiosity.

Acknowledgements. This work was supported by the Programme National Physique et Chimie du Milieu Interstellaire (PCMI) of CNRS/INSU with INC/INP co-funded by CEA and CNES. Also acknowledged is the support of COST Action CM0805 (The Chemical Cosmos).

\section{References}

Albert, S., Chen, Z., Keppler, K., et al. 2019, Phys. Chem. Chem. Phys., 21, 3669 Behnke, M., Medvedev, I., Winnewisser, M., De Lucia, F. C., \& Herbst, E. 2004 ApJS, 152, 101

Belloche, A., Garrod, R. T., Müller, H. S. P., \& Menten, K. M. 2014, Science, 345,1584

Bergantini, A., Abplanalp, M. J., Pokhilko, P., et al. 2018, ApJ, 860, 108

Bertin, M., Doronin, M., Fillion, J. H., et al. 2017, A\&A, 598, A18

Boys, S. F., \& Bernardi, F. 1970, Mol. Phys., 19, 553

Casassa, S., Calatayud, M., Doll, K., Minot, C., \& Pisani, C. 2005, Chem. Phys. Lett., 409, 110

Collins, M. J., \& Boggs, J. E. 1972, J. Chem. Phys., 57, 3811
Combes, F., Gerin, M., Wooten, A., et al. 1987, A\&A, 180, L13

Cooper, G. W., Onwo, W. M., \& Cronin, J. R. 1992, Geochim. Cosmochim. Acta, 54, 4109

Cronin, J. R., \& Pizzarello, S. 1997, Science, 275, 95

Creswell, R. A., \& Schwendeman, R. H. 1977, J. Mol. Spectr., 64, 295

Creswell, R. A., Manor, P. J., Assink, R. A., \& Schwendeman, R. H. 1977, J. Mol. Spectr., 64, 365

Daly, A. M., Kolesniková, I., Mata, S., \& Alonso, J. L. 2014, J. Mol. Spectr., 306, 11

Dickens, J. E., Irvine, W. M., Ohishi, M., et al. 1997, ApJ, 489, 753

Engel, M. H., \& Macko, S. A. 1997, Nature, 389, 265

Frisch, M. J., Trucks, G. W., Schlegel, H., et al. 2016, Gaussian09, Revision D. 01 (Wallingford, CT: Gaussian. Inc)

Gottlieb, C. A. 1973, Molecules in the Galactic Environment, eds. M. A. Gordon, \& L. E. Snyder (New York: Wiley Interscience), 181

Grimme, S., Antony, J., Ehrlich, S., \& Krieg, H. 2010, J. Chem. Phys., 132, 154104

Groner, P., Medvedev, I. R., De Lucia, F. C., \& Drouin, B. J. 2008, J. Mol. Spectr., 251, 180

Hirose, C. 1974, ApJ, 189, L145

Hollis, J. M., Jewell, P. R., Lovas, F. J., Remijan, A., \& Möllendal, H. 2004, ApJ, 610, L21

Hollis, J. M., Remijan, A., Jewell, P. R., \& Lovas, F. J. 2006, ApJ, 642, 933

Hudson, R. L., Loefler, M. J., \& Yocum, K. M. 2017, ApJ, 835, 225

Irvine, W. M., Brown, R. D., Cragg, D. M., et al. 1988, ApJ, 335, L89

Karton, A., \& Talbi, D. 2014, Chem. Phys., 436, 22

Kelvin, M. A. 1904, Baltimore Lectures on Molecular Dynamics and the Wave Theory of Light, ed. C. J. Clay (Cambridge, UK: Cambridge University Press), 602

Kresse, G., \& Furthmüller, J. 1996, Phys. Rev. B, 54, 11169

Kresse, G., \& Hafner, J. 1994, J. Phys. Condens. Matter, 6, 8245

Kresse, G., \& Joubert, D. 1999, Phys. Rev. B, 59, 1758

Lattelais, M., Pauzat, F., Ellinger, Y., \& Ceccarelli, C. 2009, ApJ, 696, L133

Lattelais, M., Pauzat, F., Ellinger, Y., \& Ceccarelli, C. 2010a, A\&A, 519, A30

Lattelais, M., Ellinger, Y., Matrane, A., \& Guillemin, J.-C. 2010b, Phys. Chem. Chem. Phys., 12, 465

Lattelais, M., Bertin, M., Mokrane, H., et al. 2011, A\&A, 532, A12

Loison, J.-C., Agúndez, M., Marcelino, N., et al. 2016, MNRAS, 456, 4101

Lykke, J. M., Coutens, A., Jorgensen, J. K., et al. 2017, A\&A, 597, A53

Martins, Z., \& Sephton, M. A. 2009, in Origins and Synthesis of Amino Acids, ed. A. B. Hughes (Weinheim, Germany: Wiley VCH), 1, 42

McDonald, J. N., Norbury, D., \& Sheridan, J. 1978, J. Chem. Soc., Faraday Trans., 74, 1365

McGuire, B. A. 2018 ApJS, 239, 17

McGuire, B. A., Carroll, P. B., Loomis, R. A., et al. 2016, Science, 352, 1449

Melandri, S., Favero, P. G., \& Caminati, W. 1994, Chem. Phys. Lett., 223, 541

Mesko, M., Zou, L., Brandon Carroll, P., \& Widicus Weaver, S. L. 2017, J. Mol. Spectr., 335, 49

Müller, F., \& Bauder, A. 1996, J. Mol. Spectr., 179, 61

Nummelin, A., Dickens, J. E., Bergman, P., et al. 1998a, ApJ, 168, 275

Nummelin, A., Bergman, P., Hjalmarson, Å., et al. 1998b, ApJS, 117, 427

Perdew, J. P., Burke, K., \& Wang, Y. 1996, Phys. Rev. B, 54, 16533

Pizzarello, S., \& Cronin, J. R. 1998, Nature, 394, 236

Pizzarello, S., \& Cronin, J. R. 2000, Geochim. Cosmochim. Acta, 64, 329

Remijan, A., Snyder, L E., Liu, S. Y., Mehringer, D., \& Kuan, Y. J. 2002, ApJ, 576,364

Requena-Torres, M. A., Martin-Pintado, J., Rodriguez-Franco, A., et al. 2006, A\&A, 455, 971

Schuurman, M. S., Muir, S. R., Allen, W. D., \& Schaefer, III, H. F. 2004, J Chem. Phys., 120, 11586

Snyder, L. E., Buhl, D., Schwartz, P. R., et al. 1974, ApJ, 191, L79

Snyder, L. E., Lovas, F. J., Mehnringer, D. M., et al. 2002, ApJ, 578, 245

Turner, B. E., \& Apponi, A. J. 2001, ApJ, 561, L207

Zingsheim, O., Müller, H. S. P., Lewen, F., Jorgensen, J. K., \& Schlemmer, S. 2017, J. Mol. Spectr., 342, 125 


\section{Appendix A: Quantum chemistry approaches}

All the theoretical data reported in this work have been obtained by means of numerical simulations using standard approaches for both, free flyer gas-phase structures and energetics, and solid gas interactions. The structures and relative stabilities of the chiral molecules with respect to the other possible free flyers of the same chemical formula are determined by means of quantum DFT using the hybrid B3LYP and BH\&HLYP methods coupled with split-valence polarized basis sets of triple-zeta quality. Each structure, fully optimized, was verified to be a stationary point by vibrational analysis. All calculations were performed using the methods and basis sets as implemented in the Gaussian09 package (Frisch et al. 2016).

A methodology based on periodic DFT, often referred to as first principle calculations, was employed to deal with the interactions of the free flyers (Fl) with the ice surface. Such treatment has been previously used and has proved satisfactory for modeling bulk and surface ice structures (Casassa et al. 2005). With the same methods, adsorption of organic molecules (Lattelais et al. 2011; Bertin et al. 2017) on various grain surfaces have been successfully investigated. More technical details can be found in the above references. Within this framework the stabilization energies are defined as

$E_{\mathrm{Ads}}(\mathrm{Fl})=\left(E_{\text {ice }}+E(\mathrm{Fl})\right)-E$,

where $E_{\text {ice }}$ is the energy of the pristine crystalline or amorphous ice, $E(\mathrm{Fl})$ is the energy of the free flyer $\mathrm{Fl}$, and $E$ is the total energy of the $[$ ice $+\mathrm{Fl}]$ system. In this expression, all energies are calculated in the periodic context of solid-state physics, all entities being optimized in isolation.

In practice, all calculations were performed with the Vienna ab initio simulation package (VASP; Kresse \& Hafner 1994; Kresse \& Furthmüller 1996; Kresse \& Joubert 1999), a code specifically designed for studying solid-state electronic structures in a periodic formalism. The main characteristics of this code is to use a plane-wave expansion of the basis set associated with projector-augmented wave ultra-soft pseudo-potentials for the atomic cores. There is no basis set superposition error (BSSE) artifact in this formalism (Boys \& Bernardi 1970). The functional form employed is the PBE (Perdew et al. 1996) exchange-correlation functional. The contribution of long-range weak interactions was taken care of within the so-called D2 Grimme correction scheme (Grimme et al. 2010). 